\title{
Primäre Haarneubildung auf der intermarginalen Kantenfläche des Augenlides als die gewöhnliche Ursache der Trichiasis.
}

Ton

E. Raehlmann in Dorpat.

Hierzu Taf, VII-X, Fig. 1-18.

Die intermarginale Liảkantenzone gehört zu den wenigen Orten der Hautoberfläche des menschlichen Körpers, welche der Haaranlagen vollständig entbehren. Es ist diese Gegend also vergleichbar den wenigen von Unua und Anderen citirten Körperstellen, nämlich der Glans penis, dem inneren Vorhautblatte, den kleinen Labien und dem Lippenroth; um so mehr vergleichbar, als auch die Lidränder zur Zeit, wo die Haarentwiekelung der Körperoberfläche beim Embryo stattfindet, ganz wie die Vorhautblätter etc. noch mit einander verwachsen sind.

In der zwischen innerer und äusserer Lidkante ausgebreiteten Hautzone finden sich aber, wie keine Haare, so auch keine eigentlichen Talgdrüsen (Haarbalgdrüsen) vor, während an den von Unna citirten Regionen, wie auch an der Hohlhandfläche, der die Haare ebenfalls fehlen, Talgdrüsen in reichlicher Menge vorhanden sind. In der Haut der Lidkante giebt es dafür allerdings besondere Talgdrïsenorgane, die Moll'schen und Meibom'schen Drüsen, welche aber beide mit Haaren und Haarbildung nichts zu thun haben. 
Prim. Haarneubildung auf der intermarginalen Kantenfläche etc. 67

Es ist also die intermarginale Hautparthie der Lider gesundheitsgemäss als haarlos zu betrachten. Unter pathologischen Verhältnissen beobachtet man indess gar nicht selten auf der genannten Stelle abnorme Haarbildung, welche unter dem Namen Distichiasis resp. Trichiasis ein wohl charakterisirtes Krankheitsbild abgiebt und von eben so charakteristischen entzündlichen Folgeerscheinungen an Lid und Auge, namentlich an dessen Hornhaut begleitet ist.

Die Namen Distichiasis und Trichiasis werden gegenwärtig von vielen Autoren promiscue gebraucht. Uxsprünglich bezeichnet der Name Distichiasis eine überzählige Cilienreihe. Der Ausdruck: Trichiasis wurde für schief nach Innen gerichtete Haare gebraucht. Da die Haare der überzähligen Cilienreihe aber häufig auch schiefe Richtung zeigen, ist Distichiasis häufig mit Trichiasis gepaart.

Unter ganz normalen Verhältnissen und an gesunden Lidern findet man aber bis zu vier Reihen Cilien dicht hinter einander auf der vorderen Kante des Lidrandes. Manche Autoren rechnen auch solche Zustände zur Distichiasis. Andere sprechen von Distichiasis nur dann, wenn die überzähligen Haare nicht aus der vorderen Lidkante, sondern aus der intermarginalen Fläche entspringen.

Die letztere Eigenthümlichkeit, bei welcher die Haare auch meistens schief gerichtet sind, verdient unser besonderes Interesse. Ich werde sie in der Folge ausschliesslich als Trichiasis bezeichnen.

Wir treffen diese Anomalie sowohl unter Umständen, wenn der Lidrand, welcher die Haare trägt, seine normale Gestalt mit zwei zugeschärften Kanten behalten hat, als auch, wenn dieser Lidrand durch Entzündungs- und Schrumpfungsvorgänge stark defigurirt ist, und eigentlicher Kanten, namentlich der inneren, entbehrt.

Rücksichtlich der Ausbildung des Leidens kommen verschiedene Grade vor. In leichten Fällen steht nur ein, oder stehen einzelne Haare falsch gerichtet auf der inter- 
marginalen Lidrandzone, in anderen Fällen ist eine vollständig geschlossene, überzählige Reihe von (falschen) Haaren auf derselben vorhanden. In noch anderen Fällen ist die ganze Lidrandfläche hart bis zur inneren Kante vollkommen und dicht behaart (vergl. Taf. VII, Fig. 1).

Nach Auffassung einzelner Autoren sind die falsch gestellten Haare angeboren (Stell wag ${ }^{1}$ ) oder entwickeln sich im Kindesalter (0. Becker). Nach Anderen entstehen sie zur Zeit der Pubertät.

Gemäss der Vorstellung der meisten Autoren aber ist die Distichiasis, sowie die Trichiasis die Folge einer nar' bigen Schrumpfung des Lidkantengewebes, durch welche eine Dislocation des Haarbodens der Cilien bewirkt sein soll: Stellwag ${ }^{2}$ ), Michel ${ }^{2}$ ), Fuchs ${ }^{4}$ ) und Andere. Es ist dabei sowohl an eine Verschiebung der Haarbälge aus der normalen Richtung, als auch an eine narbige Verzerrung ihrer Ausmündungsstellen gedacht worden.

Meine eigenen Erfahrungen stimmen mit den Angaben der Autoren nicht überein.

Bei Kindern unter zehn Jahren habe ich eigentliche Trichiasis niemals angetroffen. Auch bei Leuten unter zwanzig Jahren ist der Zustand nur selten zu beobachten. Dieses Verhältniss des Auftretens spricht an sich schon gegen die Annahme, dass man es mit einem angebornen Leiden zu thun hat. Relativ häufig findet sich Trichiasis und Distichiasis bei Individuen zwischen 20 und 35 Jahren, ist dagegen bei älteren Individuen, als frisch entstandene Anomalie, wieder selten zu finden. Die Entstehung des abnormen Haarwuchses fällt also meistens in ein Lebensalter, welches auch das grösste Contingent für Trachom liefert.

1) Stellwag, Handb. der Augenheilkunde, S. 518. Wien 1870.

2) Dasselbe S. 518 .

${ }^{3}$ ) Michel, 0. J., Lebrbuch der Augenheilkunde. Wiesbaden 1884. S. 171.

4) Fucbs, Lehrbuch der Angenheilkunde 1889. S. 525. 
Prim. Haarneubildung auf der intermarginalen Kantenfläche etc. 69

Weitaus die Mehrzahl, etwa ${ }^{9 / 10}$ aller Fälle von Trichiasis, welche hierorts angetroffen werden, finden sich an trachomatös erkrankten Augen.

Dass nach Blepharitis ulcerosa, nach Variola und nach ähnlichen ulcerativen Erkrankungen der Lidränder, durch Narbenverziehung Trichiasis entstehen kann, ist bekannt. Es handelt sich dann aber meistens um Abzweigung und Einwärtsziehung einzelner Ciliengruppen, nicht um unabhängige intermarginale Haare (Pseudocilien).

Die eigentliche typische Trichiasis ist eine Folge der interstitiellen Blepharitis ciliaris, wie sie besonders nach schwerem Trachom beobachtet wird. An Augen, welche an frischem Trachom leiden, findet sich diese Anomalie noch nicht oder nur höchst selten. Im Ausgange schweren Trachoms aber stellt sich die Trichiasis mit einer Häufigkeit, man möchte sagen Regelmässigkeit ein, welche für das Abhängigkeitsverhältniss ein untrügliches Zeugniss ablegt.

Fast die Hälfte aller Patienten, welche ausgesprochene Trachomnarben mit Knorpelverdickung tragen, sind mit Trichiasis behaftet.

Germann ${ }^{2}$ ) fand unter 180 an schweren Narbentrachom leidenden Augen $92 \mathrm{mal}$, also in $51 \%$ Distichiasis resp. Trichiasis gegeben. Vorwiegend ist das obere Lid Sitz der Erkrankung.

Aus dem angeführten Abhängigkeitsverhältniss lässt sich entnehmen, dass das abnorme Wachsen der Haare anhaltende Ernährungsstörungen des Lid- namentlich des Lidrandgewebes voraussetzt. Obwohl nun nach Trachom vielfach Narbenbildungen innerhalb des Lidrandgewebes vorkommen, habe ich mich doch überzeugt, dass sie direct zu dem abnormen Haarwachsthum keine Veranlassung geben, wohl aber indirect die Richtung der bereits im Wachsthum begriffenen Haare verändern können.

1) Germann, Inaugural-Abhandlung. Dorpat 1885. 
Wie aber ist die Haarbildung selbst zu erklären?

Dass die bei Trichiasis vorkommenden Haare nicht sämmtlich aus ihrer Richtung verdrängte Cilien sein können, wie das gemeinhin behauptet wird, ist für jemanden, der sich den Lidrand bei ausgesprochener Trichiasis (Fig. 1) genau angesehen hat, ohne Weiteres klar. Es liegt nahe, an eine Neubildung von. Haarbälgen au denken, welche die schief resp. falsch stehenden Cilien herrorgehen lassen. Diese Möglichkeit ist bisher, soviel mir bekannt, nicht erörtert worden.

Die Annahme einer Haarneubildung auf einer sonst gảnzlich haarlosen Stelle, hat allerdings a priori auch wenig Wahrscheinlichkeit für sich. - Indess ist leicht zu beweisen, dass es sich in der That bei typischer Trichiasis um eine wirkliche Hypertrichosis handelt.

Rücksichtlich der Herkunft der neuen Haare wären zwei Möglichkeiten ins Auge zu fassen. Es können die neu angelegten Haarbälge von den Bälgen der eigentlichen Cilien durch Sprossenbildung abgezweigt sein (secundäre Haarbildung) oder aber sie könnten unabhängig von den Cilien sich entwickelt haben und müssen in diesem Falle von der Haut der Lidkante entstanden sein (primäre Haarbildung) (Goette).

In der ophthalmologischen Literatur fehlen bezügliche Angaben voliständig und es ist, soviel mir bekannt, bisher kein Versuch gemacht worden, die Entwickelung der ,filschen Cilien" histogenetisch zu verfolgen.

Die einzige Angabe über die Fntstehung der falschen Wimperhaare findet sich bei Unna ${ }^{1}$ ). "Andererseits treibt an den Cilien gerade das wuchernde Haarbeet oft horizontal liegende Epithelzapfen in die Cutis, die zur Grundlage schiefstehender Cilien werden können (Trichiasis, Distichiasis)."

*) P. G. Unna in v. Ziemssen's Handb, der speciellen Pathologie und Therapie, Bd. XIY, S. 77. 
Prim. Haarneubildung auf der intermarginalen Kantenfläche etc. 71

Nach dieser Auffassung wären die schiefgerichteten Haare in Folge secundärer Bildung aus den Cilien entstanden.

Diese Unna'sche Erklärung ist, wie es scheint, von der praktischen Ophthalmologie weniger, als sie verdient, beachtet worden; sie kann sich aber offenbar nur auf solche falsch gerichteten Cilien beziehen, welche aus den Haarbälgen der ursprünglich gesunden Cilien hervorwachsen, und darum eine falsche Richtung zeigen, weil ihnen solche durch ihre schiefe Umbiegung aus dem Zapfen in den ursprünglichen Balg angewiesen wird. So habe ich die Unna'sche kurze, oben angeführte Bemerkung gedeutet.

Ich selbst habe an den Bälgen der echten Cilien bei vorhandener Distichiasis zwar keine genau oder annähernd horizontale Sprossen gefunden, wohl aber öfter zwei Cilienhaarbälge, welche in der Cutis mit ihren Wurzelscheiden vollkommen getrennt neben einander lagen, nach unten convergirten und in einen gemeinsamen unteren Theil mündeten, angetroffen. Beide Haare traten aus derselben Oeffnung an der vorderen Lidkante hervor. Das eine war normal, wie die Cilien, das andere schief gerichtet. Bisweilen war das seitlich in einen senkrecht zur Lidkante gerichteten Haarbalg einmündende. Haar nicht normal entwickelt und zeigte Abnormitäten, auf welche ich anderen Ortes zurückkommen werde.

Ich nehme demnach keinen Anstand, für diese Form der Distichiasis, soweit es sich um schief stehende Cilien der vorderen Lidkante handelt, die Unna'sche Erklärung für zutreffend zu halten, vermag sie aber nicht anzuwenden auf die Entstehung der Haare, welche am falschen Orte (oft in ganz gerader Richtung) entfernt von der vorderen Kante hervorwachsen.

Eine grosse Reihe histologischer Präparate aus dem Lidrande von an Trichiasis leidenden Personen hat mir ergeben, dass in solchen Fällen die falschen intermarginal 
hervorwachsenden Haare aus Bälgen stammen, welche von der Lidrandfläche aus durch Epithelsprossenbildung nea angelegt sind. Serienschnitte durch geeignete Theile der Lidkante gelegt, ergeben, dass die Neubildung der Haarsprossen nach embryonalem Typus vor sich geht, wobei aber einzelne unten näher zu erörternde Abweichungen von den embryonalen Haaranlagen zu constatiren sind.

Der Nachweis der primären Haarbildung bei Lidrandtrichiasis ist daher nicht allein ophthalmologisch, sondern auch entwickelungsgeschichtlich von Interesse, insofern als er lehrt, dass eine postfoetale Haarbildung von der Oberhaut aus, welche von vielen Histologen geleugnet wird, wirklich vorkommt.

Die Beobachtung einer primåren Haarentwickelung am Lidrande steht also im Einklang mit den Angaben Goette's ${ }^{1}$ ) and Werthheim's ${ }^{2}$ ) über Haarbildung überhaupt. Stieda ${ }^{3}$ ) lässt wie $\mathrm{Henle}^{4}$ ) die Frage nach einer postfoetalen Haarbildung unentschieden. Kölliker ${ }^{5}$ ), welcher diese Frage ebenfalls offen liess, hat sich jüngst ${ }^{6}$ ) mit Entschiedenheit für eine Noubildung von Haaren von der Epidermis der Kopfhaut des Menschen aus ausgesprochen; ebenso Hesse ${ }^{7}$ ).

Unnas $\left.{ }^{8}\right)$ leitet alle Haarbildung ron der mittleren Balgregion (Unna's Haarbeet) der alten Haarbälge ab. "Ohne voranfgehende Beethaare entstehen beim Erwachsenen, wenigstens nach der Pubertät, überhaupt Keine jungen Papillenhaare mehr."

1) Goette, Milroskopische Anatomie. Bd. II, S. $286^{\circ}$.

3) Werthheim, Deber den Bau des Haarbalges beim Menschen. Wiener akadem. Sitzungsbericht 1864.

3) L. Stieda, Biol. Centralblatt. VII. Bd.

4) Henle, Handbuch der systemat. Anatomie des Menschen. Bd. II, S. 23. 1873.

5) Kölliker, Mikroskopische Anatomie. Bd. II, S. 151.

6) Kölliker, Handb. der Gewebelehre des Menschen. Bd. I, S. 285. Leipzig 1889.

7) Hesse, Zeitschr. f. Anatomie und Entwickelungsgeschichte. Bd. I, S. 285 .

8) Unna 1. c. S. 71 . 
Prim. Haarneubildung auf der intermarginalen Kantenfäche etc. 73

Feiertag ${ }^{1}$ ) hält eine primäre $d$. h. von dem Hautepithel ausgehende Haarbildung bis zur Pubertatstsentwickelung (so lange das Individuum wächst,) für möglich; ähnlich äussern sich Frey ${ }^{2}$ ) und Waldeyer ${ }^{3}$ ).

v. Ebner ${ }^{4}$ ) stellt wieder das Vorkommen postembryonaler Haarneubildungen völlig in Abrede.

Gerade die intermarginale Lidkantenzone ist fïr die Entscheidung dieser Frage besonders wichtig, weil sie wie mehrfach erwähnt, normaliter weder Haarbalgdrüsen noch Haare besitzt.

Die Frage nach der Neubildung von Haaren hängt auch innig zusammen mit jener über die Regeneration derselben. Auch hier gehen die Ansichten auseinander.

Viele Autoren sind der Ansicht, dass das junge Haar (beim Haarwechsel) sich auf der alten Papille entwickelt (Langer ${ }^{5}$ ), Kölliker ${ }^{6}$ ), v. Ebner ${ }^{7}$ ), Strogonow ${ }^{8}$ ), Giovannini ${ }^{9}$ ).

Andere schreiben dem jungen Haarbeete eine neugebildete Papille zu (Steinlin ${ }^{10}$ ), Stieda ${ }^{11}$ ), Feiertag ${ }^{12}$ ), Redtel ${ }^{13}$ ).

Wieder Andere halten bei der Regeneration der Haare beides für möglich, so Unna ${ }^{14}$ ), Waldeyer ${ }^{15}$ ), Ranvier ${ }^{16}$ ).

1) Feiertag, J., Ueber die Bildung der Haare. Inang.-Abhandlung, Dorpat 1875.

2) Frey, Handbuch der Histologie ete. S. 408. Leipzig 1874 .

3) Waldeyer, Atlas der menschliehen und thierischen Haare. S. 38.1884 .

4) v. Ebner, Wiener akadem. Sitzungsberichte 1876 .

5) Langer, Biol. Centralbl. V.

6) Kölliker l. c. S. $244 . \quad$ 3) v. Ebner, l. c.

8) Strogonow, Centralbl. für medic. Wissensch. 1889, S. 516.

$\left.{ }^{9}\right)$ Giovannini, Arch. für mikrosk. Anatomie. Bd. XXXVI, 4, S. 563.

10) Steinlin, Zeitschr. für rationelle Medicin. Bd, II.

11) L. Stieda, Biol. Centralblatt, Bd. VII.

12) Feiertag, l. c.

${ }^{13}$ ) Redtel, Zeitschr. für wissenschaftl. Zoologie von Siebold und Kölliker. Bd. XXIII. 1873.

14) Unna, 1. c. ${ }^{15}$ ) Waldeyer, l. c. S. 38.

16) Ranvier "Leçons sur les maladies de la peau" par Besnier et Doyon. Paris 1881. 
E. Raehlmann.

Gegenüber der embryonalen Haarkeimbildung von der Epidermis aus (Goette's primärer Haarbildung) würden die beim Haarwechsel anftretenden nenen Keime der secundären Haarbildung entsprechen.

Es kommt aber noch eine durch die Untersuchnngen hervorragender Autoren sichergestellte Haarbildung, deren Vorkommen indess von mancher Seite angezweifelt wird, in Betracht, welche nicht embryonale Bildung ist und mit dem Haarwechsel nichts zu thun bat, aämlich die durch Seitensprossen aus der Stachelschicht eines Haarbalges hervorgehenden seitlichen Keime, auf welche namentlich Unna ${ }^{1}$ ) die Aufmerksamkeit gelenkt hat.

Unna möchte annehmen, dass im postfoetalen Leben in der Kopfhaut die Haare auf solche Weise sich vermehren, indem die seitlichen Fortsätze sich später trennen und auf diese Weise die Haarkreise (Pincus) hervorbringen,

Nach den Bildern, die ich gesehen, wächst das Haar, an den Ciliensprossen wenigstens, ausnahmslos in der Richtung des Epithelzapfens weiter und also in den alten Balg hinein (vergleiche oben).

An den Cilien habe ich seitlich in einen geraden Haarbalg mit entwickelter Cilie einmündende, selbstständige Bälge, welche junge Haare enthielten, bei bestehender Distichiasis mehrmals angetroffen. Eine Abschnürung einer solchen, gewissermaassen selbstständig gewordenen Sprossung habe ich indess niemals gefunden. Es ist aber klar; dass bei solcher abnormen Stellung der Wurzelanlagen der Haare diese letzteren schief aus den Cilienbälgen mit der Richtung gegen den Bulbus hervorwachsen und so zu Distichiasis Veranlassung geben können. Für diese Art der Distichiasis bleibt es aber charakteristisch, dass die falsch gestellten Haare aus der vorderen Kante, wie die normalen Cilien, hervorwachsen.

Für die abnorme Behaarung der intermarginalen Zone aber, wo die Haare getrennt ron den Oeffnungen der Cilienhaarbälge aus der Lidrandfläche bisweilen dicht neben

1) Unna, 1. c. S. 77. 
Prim. Haarneubildung auf der intermarginalen Kantenfäche etc, 75

den Ausführungsgängen der Meibom'schen Drüsen hervorwachsen, können wir die secundäre Bildung ron den Cilien aus nicht verantwortlich machen.

Um die Entstehung dieser Haare zu erkennen, habe ich eine grosse Reihe anatomischer Untersuchungen ausgeführt. Es standen mir einzelne Augenlider mit Trichiasis zur Verfiugung, welche zufällig an der Leiche gefunden wurden. Das meiste Material war operativ gewonnen. Zur Beseitigung der Trichiasis wird die intermarginale Randzone mit den falsch wurzelnden Cilien ausgeschnitten und der Defect durch Transplantation eines Hautstïckes ersetzt. So habe ich ein grosses Untersuchungsmaterial gewonnen. Das letztere wurde in Alkohol gehärtet, theils in Celloidin, theils in Paraffin eingebettet und in Serienschnitte zerlegt.

Die mikroskopische Untersuchung zeigte zur Evidenz, dass die falsch zwischen innerer und äusserer Lidkante aus der Lidhaut gewachsenen Haare sämmtlich primäre Bildungen sind, welche nach embryonalem Typus entstehen.

Auf Frontal- und Sagittalschnitten durch die mit Trichiasis behaftete Lidkante trifft man Haarbildungen in den allerverschiedensten Stadien der Entwickelung an und ron der ersten primitivsten Haaranlage, den tief eingesenkten Epithelsprossen bis zur Papillenbildung, der Bildung des sogenannten primitiven Haarkegels und endlich zum ausgewachsenen Haar finden sich alle Uebergänge vor. Es lässt sich aber auch, wo solche ersten Stufen der primären Haarbildung nicht sichtbar sind, an den Schnittserien mit absoluter Sicherheit feststellen, dass man es mit selbstständigen, im Cutisgewebe isolirt eingelagerten, auf der intermarginalen Lidrandfläche ausmündenden Bälgen zu thun hat, in denen die Haare normale Wachsthumsverhältnisse und auch den normalen Haarwechsel durchmachen.

An Serienschnitten, die horizontal also parallel zur intermarginalen Kantenfläche angelegt sind, lässt sich nach- 
weisen, dass die Wurzelscheiden der Trichiasishare weder mit einander, noch auch mit denen der normalen Cilien zusammenhängen; es fehlen jegliche Epithelzellen-Communicationen (Zellenzapfen oder Sprossen), welche etwa auf die Genese der Haare aus seitlichen Ausläufern der Cilien (Unna) respective deren Wurzelscheiden schliessen liessen. Die Bälge der falschen Haare mit ihrem Inhalte sind von den Cilien durchweg in ganzer Ausdehnung getrennt. Das Stratum mucosum derselben geht direct in die Malpighi'sche Schleimschicht der Epidermis auf der Haut des Lidrandes ïber. Diese Bilder sind in allen Präparaten, welche ich durchmusterte, und welche sich auf mehr als ein Dutzend Fälle von Trichiasis beziehen, immer wieder anzutreffen, so dass die Berechtigung zu dem Rückschlusse vorliegt, dass die neu gewachsenen Haare nicht von den Cilien abstammen können.

Wenn wir diesen Schluss acceptiren, bleibt rücksichtlich der Entstehung der Haare wohl nur übrig, an eine primäre Neubildung derselben zu denken.

Es ist hier auch der Einwand nicht statthaft, dass etwa embryonal angelegte also angeborene Haare nach Art kleiner Lanugohärchen relativ spät beim Erwachsenen besondere Mächtigkeit erreichten; denn wir wissen ja, dass die Lidkantenzone jeglicher Haaranlagen entbehrt.

Den eigentlichen Beweis für die Neubildung liefern uns aber erst die anatomischen Befunde der ersten Anlagen junger Haare. Man findet solche junge Haarbildungen im Allgemeinen um so sicherer und zahlreicher, je kürzere Zeit die Trichiasis bestanden hat, also namentlich zu Beginn des Leidens, wenn die neu wachsenden Haare noch geringe Stärke besitzen. In solchen Fällen findet man neben älteren Bälgen fast immer junge eben durchbrechende Haare und häufig auch ganz junge Haarkeime.

Die Abbildung (Taf. VII, Fig. 1) giebt naturgetreu das Aussehen des Lidrandes eines Mädchens von 24 Jahren 
Prim. Haameubildung auf der intermarginalen Kantenfläche etc. 77

wieder, welches seit Jahren an Trachom gelitten hat, aber angeblich erst seit einigen Monaten an Trichiasis leidet. Der Knorpel des oberen Lides ist leicht verkrümmt, seine Innenfläche von einer dicken frischen Trachomnarbe überzogen, die Hornhaut zeigt diffusen Pannus. Der Lidrand des Oberlides ist noch gut erhalten, seine Kanten sind noch ziemlich scharf. Die vordere trägt zwei Reihen dichtstehender, kräftiger, gut gewachsener Cilien. Einwärts und hinten von den letzteren, zwischen ihnen und der inneren Lidkante, also auf der intermarginalen, in der Mitte circa $6 \mathrm{~mm}$ breiten Lidrandfläche, finden sich eine Menge kleiner papillenartig vorgewölbter Hügelchen, welche auf ihrer Kuppe eine Oeffnung erkennen lassen, aus welcher meistens ein Haar austritt. Die Oberfläche des Lidrandes zeigt wegen dieser Hügelchen ein grobgranulirtes, gänsehautähnliches Aussehen.

Diese Oberfläche ist von einer dicken Fettschicht eingeölt, welche halb flüssig zwischen und auf den Hügelchen lagert, aus deren Oeffnung sie vorquillt (Lidrandseborrhoe). Die Haare sind theils lang, theils sparrig und kurz, zeigen die verschiedensten Wachsthumsrichtungen, die dicht an der inneren Lidkante stehenden sind ganz nach einwärts gekehrt und berühren in natürlicher Lage des Lides die Hornhaut.

Die intermarginale Randparthie mit den falsch stehenden Cilien wurde durch zwei parallel den Lidkanten vom inneren zum äusseren Lidwinkel verlaufenden, nach oben convergirenden Schnitte so exstirpirt, dass vorn die Cilien, hinten die innere Lidkante mit den Ausmündungsstellen der Meibom'schen Drüsen stehen bleiben, die Wurzeltheile der intermarginalen Haare aber mit dem keilförmig prismatisch umschnittenen Stück entfernt wurden.

Dieses letztere Stück wurde in Alkohol gehärtet, in toto in Boraxcarmin gefärbt und in Paraffin eingebettet.

Die von diesem Präparate gewonnenen Schnittserien 
gehen frontal durch das Gewebe. In der Serie fehlt kein Schnitt, jeder ist 10 Micra dick. Die Schnitte zeigen neben erwachsenen, vollentwickelten Haaren, Papillenhaaren, auch Kolbenhaare (Beethaare Unna's), letztere in verschiedener Höhe des Haarbalges steckend. Dann aber finden sich primäre Haaranlagen, einfache, solide tief in die Cutis eindringende Zapfen, welche theils cylinderformig, theils kolbenförmig mit abgerundeten Enden scharf gegen das subcutane Kantengewebe sich abgrenzen und im normalen Lidkantengewebe stets fehlen. Die Zapfon zeigen in der peripheren Mantelschicht eine einfache Lage typischer Cylinderzellen; gleich oberhalb derselben liegen die den Zapfen gänzlich ausfüllenden Stachelzellen. In einzelnen dieser Zapfen ist am unteren Ende eine Einschmelzung des Lumens, in anderen eine seitliche solide Sprossung bemerkbar, offenbar Talgdrüsenanlagen.

Eine spätere Phase der Haarentwickelung wird repräsentirt durch die Bildung der Papille und des Balggewebes. Aber, und das ist ein Punkt, in welchem diese postembryonale Haarbildung von der typischen Embryonalen abweicht, die Papille ist schon in den Haaranlagen voll entwickelt, zu einer Zeit, wo der primitive Haarkegol noch nicht zur Entwickelung gekommen ist. Die Zellen des soliden Fortsatzes liegen noch als regelmässige Stachelschichte neben einander.

Dann finden sich Präparate, die ein weiteres Stadium der Haarentwickelung repräsentiren. Die Papillenformation ist gegeben; das Ende des soliden Zapfens kappenförmig ausgehöhlt (eingestiilpt), unterhalb der Papille sind die Zellen des Zapfens nun der Längsrichtung nach geordnet, meist oblong, einzelne spindelig gestaltet. Dadurch kommt eine Streifung zu Stande, welche im oberen (Papillen)ende des Epithelzapfens zuerst auftritt.

Die Figuren 2-12 geben das Aussehen einer solchen Haaranlage in einer Serie ron 13 Schnitten wieder. 
Prim. Haarneubildung auf der intermarginalen Kantenfläche etc. 79

Die Schnittebene geht nicht durch die Achse des Zapfens, sondern durchsetzt dieselbe schief. In Fig. $2-8$ ist der Kolben, erst in den letzten Schnitten, in Fig. 9-12, ist die Papille enthalten. Fig. 6 trifft die Mitte (Acbse) der tiefsten Theile der Haarwurzel zunächst der Papille. Hier ist die erwähnte Streifung gut zu erkennen.

Die Schnitte Figuren 6-10 enthalten die Haaranlage unterhalb der Papille, man erkennt in diesem oberen Theil des soliden Epithelzapfens zwei verschiedene Zellformen, cylindrische, einschichtig den Mantel des Zapfens bildend, und oblonge, den ganzen Inhalt herstellend. Ein eigentlicher Kegel, als primitive Haaranlage, entsprechend dem analogen embryonalen Stadium der Haarentwickelung, fehlt hier noch vollkommen. Es ist leicht, sich davon zu überzeugen, dass das ganze Zapfenende bis etwa 1/5 seiner Längenausdehnung abwärts, d.h. gegen die Hautoberfäche $z u$, innerhalb des einschichtigen Cylinderepithelmantels von oblongen. Zellen ausgefitllt ist, welche sämmtlich der Achse des Cylinders annähernd parallel laufen. Fig. 8 repräsentirt einen Schnitt, der ungefähr durch die Mitte des Zapfenhalses, unterhalb der Papille hindureh geht. Fig. 14 giebt den in Betracht kommenden Schnitttheil bei stärkerer Vergrösserung wieder, die oblongen bis spindeligen Zellkörper gehen weiter abwärts, am unteren Theil des Zapfens, in typische kubische Stachelzellen über. Der Uebergang geschieht, obne scharfe Grenze, allmälig. Soweit aber die Schichte der oblongen Zellen reicht, füllt sie den Zapfen ganz aus, von einer kegelförmigen Anordnung: Reissner ${ }^{1}$ ), Kölliker ${ }^{2}$ ), Goette ${ }^{3}$, Ebner ${ }^{4}$ ), Unna ${ }^{5}$ ), Hertwig $^{6}$ ) ist noch nichts $z \mathfrak{u}$ sehen, ebensowenig von einer

1) Reissner, Beiträge zur Kenntniss der Haare des Menschen und der Sängethiere. Breslan 1854. 2) Kölliker, l.c.

3) Goette, 1. c. 5) Eibner, l. c. 5) Unna, 1. c. S. 52.

5) Hertwig, Lehrbuch der Entwickelungsgeschichte des Menschen und der Wirbelthiere. 
flaschenförmigen Configuration der Zellen Remy's ${ }^{1}$ ). Eher gleichen die Bilder meiner Präparate der ersten Phase der Haarentwickelung, wie Giovannini ${ }^{2}$ ) sie beschreibt (premier rudiment du germe pileux).

Obwohl ich eine deutliche Längsstreifung durch Zellgruppirung in meinen Präparaten antreffe, vermisse ich eine eigentliche axiale Anordnung derselben in Säulenform, wie Ranvier ${ }^{3}$ ) sie von Embryonen beschreibt.

Nach Unna ${ }^{4}$ ) ist beim embryonalen Haarkeim, sobald die Papille entwickelt ist, anch der Haarkegel gegeben. Nach den Angaben Giovannini's ${ }^{5}$ ) findet Aehnliches auch beim normalen Haarwechsel statt, wenn sich auf der alten Papille der nene Keim entwickelt.

Auffallend ist ferner, dass bei dieser pathologischen Haarbildung im Lidkantengewebe der eigentliche Haarbalg sehr frühzeitig angelegt ist, zu einer Zeit, wo die einzelnen Haargebilde als solche noch nicht differenzirt sind. In Figur 14 sieht man eine schon gut ausgebildete Rings- und Längsfaserschichte um den jungen Haarspross herum entwickelt. Dieser Haarbalg steht von dem Epithelzapfen an dessen Kolbenende um einen Raum (Fig. 13b und Fig. 14a) ab, welcher auch bei den stärksten Vergrösserungen vollkommen homogen aussieht. Es ist wohl wahrscheinlich, dass dieser Raum, welcher längs der Haaranlage nach unten enger wird, indem die Balgscheide sich dem Zapfen mehr anlegt, beim Wachsen des jungen Haares durch Volumszunahme des Kolbens verloren geht, resp. verbraucht wird. Thatsächlich schaltet sich später zwischen Balg und Kolben die äussere Glashaut der äusseren Wurzelscheide ein, von welcher in den fraglichen Schnitten jedoch noch keine Spur aufzufinden ist.

Bemerkenswerth ist auch, dass dieselbe lichte Zone sich auch zwischen dem äussersten Haarwurzeltheile der Haarzwiebel und der jungen Papille (Figg. 14c, 10 und $11 \mathrm{~b}, 12 \mathrm{a}, \mathrm{b}$ ) findet. Es existirt in allen meinen Präparaten zwischen Papillenober-

$\left.{ }^{1}\right)$ Remy, Recherches histologiques sur l'anatomie normale de la pean etc. Paris 1878.

2) Giovannini, l. c. S. 558. 3) Ranvier, I. c. S. 178.

4) Unna, 1. c. S. 52.

5) Giorannini, D. S., De la régénération des poils après l'epilation: Arch. für mikroskopische Anatomie XXXYI, 4, S. 538. 
Prim. Haarneubildung auf der intermarginalen Kantenfläche etc. 81

fläche und dem äussersten Wurzelende des Haares ein relativ breiter Schaltraum (Fig. 10 und 11 a, Fig. 12 a d, Fig. 13 a), welcher mit der erwähnten homogenen Schichte (Fig. 14a, 13b) zwischen Haarbalg und Zellkolben (Haarzwiebel) direct zusammenhängt. Figur 13 giebt eine stärkere Vergrösserung des oberen Theils der Fig. 11 (Papille $c$ und Haarzwiebel a) wieder. An ganz jungen Haaranlagen gewinnt man den Eindruck, als handle es sich bei der Bildung der Haarzwiebel nicht um einen Einstülpungsvorgang, sondern vielmehr um ein selbstständiges Wachsthum des Fundus des Epithelzapfens, bei welchem die peripheren Mantelschichten des letzteren kappenförmig eine Zellenschicht, die junge Papille, umwachsen.

Noch eine weitere Abweichung vom embryonalen Entwickelungstypus ist bei dieser pathologischen postembryonalen Haarbildung zu beobachten.

Beim Embryo entstehen nämlich die Talgdrüsen erst zu einer Zeit (zwischen dem vierten und fünften Fötalmonate), wo der Haarkeim schon entwickelt ist. Die Differenzirung der Epithelzellen im Innern der Drüsenkolben und die Talgumwandlung erfolgt nach Kölliker ${ }^{1}$ ) erst zur Zeit, wo das junge Haar durchbricht. Nach Schulin ${ }^{2}$ ) dagegen tritt eine Verfettung der mittelsten Zellen des Talgdrüsenwulstes ein, noch ehe die erste Andeutung eines axialen Haarkegels vorhanden ist. Ganz im Einklange hiermit ist an unseren Abbildungen Fig. 2-11 zu erlsennen, dass die Drüsenbildung bereits vor Anlage des eigentlichen Haares erfolgt ist. Figg. 4, 5 und 6 lassen bereits Umwandlung der Epithelzellen behufs Secretbildung erkennen, die Drüsenkolben haben auch bereits seitliche Sprossen getrieben (Figg. 2 und 3), in welchen ebenfalls eine Umwandlung der Zellen erkennbar.

In einzelnen Pääparaten habe ich übrigens Epithelsprossen aus dem Lidkantenhautepithel mit Drüsenzellen-

1) Kölliker, Handbuch der Gewebelehre 1889, S. 268.

2) Schulin, Zeitschr. f. Anatomie und Entwickelungsgeschichte. II. Band.

v. Graefe's Archiv für Ophthalmologie. XXXVII. 2. 
umwandlung angetroffen, ohne dass ich Haaranlagen an denselben, abgesehen von soliden Epithelfortsätzen ohne Pigment und Papillenbildung auffinden konnte. Es wäre nicht unmöglich, dass sich von der Epithelwand solcher primären Talgdrüsen aus secundäre Haarkeime durch Sprossenbildung entwickelten; doch habe ich fur diese Annahme keine weiteren Belege, als die angegebenen gefunden.

Die Neubildung von Haaren nach dem beschriebenen Typus ist bei Trichiasis leicht festzustellen. Es lässt sich schon durch periodisch wiederholte klinische Untersuchung der Lidränder von an Trachom leidenden Patienten nachweisen, dass die Anomalie langsam aus unscheinbaren Anfängen entsteht. $\mathrm{Zu}$ diesen Beobachtungen empfiehlt sich die binoculare Lupe von Westien. Man findet anfangs, wenn die ersten Beschwerden den Patienten zwingen, sich dem Arzte vorzustellen, nur wenige, kleine Härchen; nach verhältnissmässig kurzer Zeit, nach Wochen, Monaten, sind dieselben lang und sparrig geworden, zwischen ihnen sind einige oder auch zahlreiche neue Haare entstanden, an Stellen, wo bei der ersten Untersuchung keine waren.

Schneidet man an solchen Lidern die intermarginale Zone mit den Trichiasishaaren aus, so findet man neben völlig erwachsenen Haaren häufig auch die beschriebenen primitiven Bildungen.

An den ausgewachsenen Haaren kann man alle Stadien des Haarwechsels vorfinden. Es finden sich Papillenhaare wie Kolbenhaare, letztere in den verschiedenen Stadien ihres Aufsteigens im Haarbalge häufig auch in jener charakteristischen Lagerung in der mittleren Balgregion, welche Unna yon seinen Beethaaren beschreibt.

Ich habe in diesen neu entstandenen Bälgen Kolbenhaar und Papillenhaar zugleich angetroffen. Es darf also angenommen werden, dass die pathologisch neugebildeten Haare ganz wie die normalen Haare anderer Körperstellen, 
Prim. Haarnenbildung auf der intermarginalen Kantenfläche etc. 83

deren Bälge aus der Embryonalzeit stammen, wachsen und wechseln.

Die Bälge der neu entstandenen Haare reichen verschieden tief in das Cutisgewebe resp. Unterhautzellgewebe hinein (vgl. Fig. 17), im Allgemeinen um so tiefer, je derber und länger die Haare sind. Man findet aber stets neben recht langen stattlichen Haaren, welche die Mächtigkeit und Länge normaler Cilien erreichen, auch schwache zarte Härchen, welche den Lanugohaaren gleichen, welche kein Mark besitzen, deren Bälge nicht tief reichen und meist noch in der Cutis sitzen.

Auf Horizontalschnitten, welche die Haaranlagen im Allgemeinen quer treffen müssen, kann man sich bei ausgesprochener Trichiasis (etwa wie in Fig. 1) davon überzeugen, dass die Anzahl der Haarquerschnitte immer mehr zunimmt, je mehr der Schnitt sich dem Lidrande nähert.

An Frontalschnitten erhält man den besten Aufschluss über die Richtung der Haarbälge und damit auch der Haare selbst.

Während die Bälge der normalen Cilien in typischer Lage eingepflanzt sind, derart, dass alle Bälge nahezu senkrecht zum Lidrande in einer Ebene gruppirt sind, oder so, dass diese Ebene einen bestimmten Kleinen Neigungswinkel gegen die Lidrandfläche einhält, zeigen die Trichiasishaarbälge die verschiedenste Richtung. Man erhält auf manchen Frontalschnitten daher Quer-, Längs- und Schiefschnitte solcher Haarbälge nebeneinander (Fig. 17). An der freien Lidrandfläche treten die Haare daher auch in der allerverschiedensten Richtung aus.

Ich habe Fälle gesehen, wo zahlreiche ,falsche" Haare auf der Randfläche vorhanden waren und die Beschwerden des Patienten gering, weil sämmtliche Haare die Tendenz zeigten, vom Bulbus abgewandt nach aussen zu wachsen und wieder andere, wo wenige Haare existirten,- dabei aber ausgedehnter traumatischer Pannus vorhanden war, weil 
die sämmtlichen Haare nach imnen gegen den Augapfel gewachsen waren.

Interessant ist die Richtung des Verlaufs der Trichiasisbälge im Lidgewebe. Mitunter ist die Richtung des Haarbalgs eine völlig gerade, so dass nur die abnorme Stelle ihrer Implantirung die "falschen" Haare von den wahren Cilien unterscheidet. In anderen Fällen beschreibt der Balg eine Curve, welche die verschiedenste Gestalt zeigen kann. Man findet Haarbälge, deren Richtung säbelförmig bis bogenförmig angelegt ist, andere, dio mehr weniger wellig gekrümmt erscheinen (Fig. 17b). In einer Schnittserie fand ich eine junge Haaranlage, einen Epithelzapfen, welcher bogenförmig eine Moll'sche Drüse umwachsen hatte, der letzteren so $\mathrm{zu}$ sagen ausgewichen war. Uebrigens hat Unna ${ }^{2}$ etwas ähnliches an den secundären Anlagen der embryonalen Achselhaare beobachtet, "letztere wachsen nach dem Orte des geringsten Widerstandes, oft auf krummem Wege mit Umgehung der im Wege liegenden Talgdrüse."

Die grösste Unregelmässigkeit lässt sich aber an der Haarwurzel beobachten. Hier fand ich häufig ein gänzliches Umbiegen des Wurzelhalses aus der ursprünglichen Richtung. in die entgegengesetzte, so dass das Haar am Halse seiner Wurzel (dicht unterhalb der Zwiebel) wie umgeknickt aussah (vgl. Fig. 15) oder die Haarwurzel machte in Gestalt eines kurzen Halbkreises eine Umbiegung zur Seite oder nach unten so, dass Papille und Haarwurzel retortenförmige Gestaltung zeigten.

In einem meiner Präparate findet sich ein sehr tief aus dem Unterhautzellgewebe kommendes Haar, dessen $\mathrm{Pa}-$ pille und Bulbus ganz nach oben und einwärts gerichtet sind (Fig. 16). Die genannten Theile liegen dicht vor der vorderen Lidknorpelfäche zwischen dieser und den Orbicularisbündeln. Das aus dieser Wurzel hervorgehende Haar

1) Uana 1. c. S. 54 . 
Prim. Haarneubildung auf der intermarginalen Kantenfläche etc. 85

macht sammt seinen Wurzelscheiden eine halbkreisförmige Drehung, durch welche das Haar die Wachsthumsrichtung nach unten gewinnt. Der convexe Rand der bogenförmigen Drehung der Haarwurzel ist gegen den Lidknorpel gerichtet und ron dessen Vorderfläche durch einige Orbicularisbündel (Fig. 16g) getrennt, das abwärts strebende Haar wächst indess von der Biegung an völlig gerade weiter und zur Kantenfläche hinaus.

Besonderen Einfluss auf die Richtung der neugebildeten Haarbälge haben narbige Veränderungen des interstitiellen Gewebes der Lidkante. Ich finde in einzelnen Schnitten mehrere Haare, welche von stark sclerosirten narbenähnlichen Gewebsziigen, welche fast immer in longitudinaler Richtung parallel zur Kante den Lidrand durchsetzen, aus ihrer Richtung verdrängt sind und dadurch einen abnormen Verlauf gewinnen.

In Fig. 15 findet sich dicht unterhalb des Halses der Haarwurzel ein derbes, entzündlich neugebildetes, sclerosirtes Gewebe (Fig. 15d), oberhalb welches der Haarbalg eine Abbiegung aus der Richtung erleidet. Der Haarbalg, schon in seiner Wurzelanlage Fig. $15 \mathrm{~b}$ stark gekrümmt, weicht durch eine neue Biegung dem Narbengewebe aus.

Am häufigsten ist dieser Verlauf so, dass der untere Theil des Haarbalges von der Haut zu der Narbe gerade aufsteigt, dann aber aus der Richtung mehr oder weniger scharf abbiegt, wobei man den Eindruck erhält, dass die erwähnte Narbe früher da war, als der Haarbalg, dass letzterer bei seinem Entstehen am Orte der Narbe auf Wachsthumshindernisse stiess und daher an der Narbe vorbei weiter gewachsen ist.

Nachdem wir gesehen haben, dass die Trichiasis auf primärer Neubildung von Haaren beruht, drängt sich die Frage auf, durch welche Veränderung des Lidrandgewebes diese Haarbildung veranlasst wird.

Es wurde oben S. 69 bereits hervorgehoben, dass wir 
Trichiasis bisweilen nach langer verschleppter Blepharitis ciliaris, am häufigsten aber bei derjenigen Blepharitis marginalis antreffen, welche im Ausgangsstadium des schweren Trachoms fasst nie vermisst wird. Bei anderen Krankheiten des Lidrandes wird diese Anomalie ungleich viel seltener beobachtet.

Die Veränderungen des Lidrandes bei Trachom sind bisher histologisch wenig beobachtet worden. Um dieselben in ihrer Entwickelung zu übersehell, ist es nöthig, die Gefässvertheilung im Lidkantengewebe zu berücksichtigen.

Die Verhältnisse, auf die es hier ankommt, sind in der vortrefflichen Abhandlung über die Lidgefässe von Fuchs ausführlich behandelt. Auf einige Besonderheiten, welche ich an Injectionspräparaten fand, mache ich in nachstchendem aufmerksam (vergl. Fig. 18).

Vor dem oberen und unteren Tarsusrande verlaufen zwei Gefässbögen, welche auf Querschnitten quer getroffen werden (Arcus tarseus superior und inferior, Fuchs) (vgl. Fig. 18, 1 arcus inf.). Von dem unteren Gefässbogen gehen vor der vorderen Knorpelfäche zahlreiche stärkere Aeste, von denen auf jedem Querschnitte (Sagittalschnitte) des Lides mehrere getroffen werden, zum Lidrande hinab. Ich möchte diese absteigenden Stämme als rami descendentes (Fig. 18, 2) besonders bezeichnen. Sie bilden durch zahlreiche Verzweigung hart oberhalb des Lidrandes in der Cutis ein Gefässlager (3), welches längs der ganzen Randfläche des Lides von der äusseren zur inneren Lidkante reicht und aus welchem über der inneren Lidkante die Conjunctivalgefässe der unteren Hälfte der Knorpelconjunctiva direct hervorgehen (Fig. 18, 4). - Dasselbe Gefässlager liefert ein relativ mächtiges Schlingennetz für die Cutispapillen des Lidrandes (Fig. 18,3) und für die Pseudopapillen der Conjunctiva (Fig. 18,4).

Die Conjunctivalgefässe der unteren Hälfte der Knorpelconjunctiva stehen in keiner Communication mit den 
Prim. Haarneubildung auf der intermarginalen Kantenfläche etc. 87

Knorpelgefässen. Perforirende Aeste des Arcus tarseus finde ich nur höher oben und zwar nur recht dünne. Die Hauptgefässe des oberen Drittels der Knorpelconjunctiva kommen von Aesten des Arcus tarseus superior und biegen um den convexen oberen Knorpelrand zur Conjunctiva hinab (Fig. 18, 10). Die Conjunctivalgefässe sind also ziemlich unabhängig von den tiefen Knorpel- und Lidgefässen. Die Gefässe des Lidrandes aber, die Fortsetzung der conjunctivalen Gefässe, communiciren zwar mit den tieferen Lidgefässen und denen des Knorpels in reichlicherer Weise, aber doch immer so, dass die Conjunctivalen und Lidrandpapillengefässe bis zur vorderen Lidkante hin ein einheitliches Gefässlager bilden. Auch nach Fuchs sind der freie Lidrand und die Conjunctiva am gefässreichsten. Die Papillengefässe des Lidrandes (3) stehen oberhalb der vorderen Lidkante in Verbindung mit den Lidhautgefässen (8). Das Capillarschlingennetz dieser letzteren tritt indess sichtlich zurück gegenüber dem reich ausgebildeten Netze der Cutispapillen des Lidrandes (3).

Das Gefässnetz (11), welches die Wurzeltheile der Cilien ernährt, hängt mit diesen Gefässen der Cutispapillen des Lidrandes gar nicht zusammen. Speciell zu erwähnen wäre, dass dieses letztere, sowie die Gefässschlinge, welche in die Papille der Cilien (a) eintritt, der Regel nach aus einer Arteria praetarsealis, Fig. 18,6, hervorgeht. In Fig. 18,11 hängt sie direct mit dem Capillarnetz der Orbicularisbündel zusammen.

Es ist aus dieser Gefässordnung ohne Weiteres klar, dass chronische Entzündungen, die mit starker Hyperämie der Conjunctivalgefässe einhergehen, einen besonders directen Einfluss auf die Ernährung des Lidrandes haben müssen, dass ferner Entzündungen mit proliferirendem Charakter weit frühzeitiger den Lidrand als den Knorpel betheiligen werden.

Umgekehrt wird der Wurzelboden der Cilien bei tiefen 
Erkrankungen des Knorpels und bei interstitieller Blepharitis frühzeitiger in Mitleidenschaft gezogen werden, bei Conjunctival- und Lidrandentzündung dagegen lange unbetheiligt bleiben können. So verhält es sich auch bei Trachom, so lange keine Tarsitis auftritt. In Fällen wie Fig. 1 (S. 77) sind die Cilien in zwei Reihen vollkommen normal goblieben; dagegen ist der Lidrand hochgradig verändert.

Bei schwerem Trachom liegen die Verhältnisse für eine Betheiligung des Lidrandgewebes besonders günstig.

Eine Stauungshyperämie wird hier in den Lidrandgefässen niemals vermisst. Daneben fand ich zahlreiche Neubildungen von Capillaren aus den Catispapillengefässen direct hervorgehend und ferner eine beträchtliche Einlagerung von Rundzellen vornehmlich und immer zuerst in der Cutiszone der Lidkante rings um die Epitheleinsenkungen (Fig. 17a). Die Infiltration mit Eiterzellen ist stellenweise dichter, stellenweise schwächer, sie reicht längs den Epitheleinsenkungen der Moll'schen Drüsen ziemlich weit hinauf, ist sonst wesentlich auf das Cutisgewebe beschränkt (Fig. 17a).

Diese Blepharitis marginalis ist im zweiten Stadium des Trachoms regelmässig vorhanden; klinisch zeigt sich der Lidrand geröthet und verdickt.

Zur Zeit, wo die eigentliche trachomatöse Conjunctivitis bereits abgelaufen ist, geht die Entzündung des Lidrandes noch weiter fort. Dieselbe trägt, wie die Conjunctivitis, proliferirenden Charakter. Es finden sich zu einer späteren Zeit der Blepharitis im Cutis- und auch spärlicher im subcutanen Gewebe grosse Mengen junger Spindelzellen, welche zur Bindegewebsbildung führen. So entsteht ein der Sklerose hochgradig verfallendes Narbengewebe, welches mit der schrumpfenden Conjunctiva und mit dem Knorpel zusammenhängt.

Zur Zeit, wo sich dieses Gewebe ausbildet, ist stets eine bestimmte Veränderung der Lidrandhaut und ihres 
Prim. Haarneubildung auf der intermarginalen Kantenfläche etc. 89

Epithels nachweisbar. Die Epithellagen sind verdickt, namentlich durch Zunahme der verhornten Schichten der Oberfläche. Es findet eine vermehrte Abstossung derselben statt. Namentlich aber vergrössern sich die Papillen der intermarginalen Zone.

Noch später kommt, wie viele meiner Präparate zeigen, entschieden eine Vermehrung der Papillen vor, dieselben werden stellenweise filiform, wachsen schief, erhalten seitliche Sprossen u. s.w.

So lange diese Veränderungen sich innerhalb gewisser Grenzen halten, ist von Haarbildung noch nichts $\mathrm{zu}$ sehen. Zwischen diesen Bildern und denen bei typischer Trichiasis scheinen indess nur graduelle Unterschiede zu existiren. Wie die entzündliche Proliferation des Epithellagers neue Papillenbildung hervorbringt, ist histologisch nicht $\mathrm{zu}$ erkennen. Es liegt hier indess eine Analogie vor mit ähnlichen Vorgängen in der trachomatösen Conjunctiva. Hier finden, wie Berlin und Iwanow zuerst zeigten, recht häufig pathologische Epithelwucherungen statt, die in Form von Zapfen und Sprossen ins veränderte Schleimhautgewebe hineinwuchern. Wir sehen, dass rom Lidrande aus bei derselben Krankheit, bei Trachom, etwas Aehnliches geschieht. Hier kommt aber noch die Haarbildung hinzu, welche als weitere Phase in der Entwickelung der Epithelwucherung betrachtet werden mag.

Wir kommen auf Grund der klinischen Beobachtung sowohl, als der histologischen Untersuchung zu dem Schlusse, dass lange anhaltende Hyperämien der Lidkantengefässe eine Proliferation des Epithelstratums (des Stratum Malpighi) herbeiführen, welche auf einer gewissen Höhe entwickelt, zur Neubildung ron Haaren und Talgdrüsen Veranlassung geben kann und häufig giebt. 


\section{Erklärung der Abbildungen auf Tafel VII - X.}

Fig. 1. Trichiasis des Oberlides. Zwei Reihen gut entwickelter: normaler Cilien. Hypertrichosis des Lidrandes nach Trachom der Bindehaut.

Fig. 2-12 (Hartnack Obj. 4 Oc. 3). Serie von 13 Schnitten schief durch eine junge Haaranlage in Fig. 2-6: interer Theil mit Talgdräse. Figuren 7-10: Bulbus (Anlage der Haarzwiebel). Fig. $10-12 a, b$ und $e$ : enthält dỉe junge Papille.

Fig. 13. Papille und Haarzwiebel von Figur 11 stark vergrössert: c Papille, a lichte Zone zwischen ihr und dem Bulbus des Epithelzapfens, $b$ lichte Zone um die Haarzwiebel.

Fig. 14. Mitte des Endes des Epithelzapfens oberhalb der Zwiebel. Starke Vergrösserung des oberen Theiles von Fig. 8. $c$ Rand der Papille, a lichte Zone um das obere Zapfenende, $b$ Pigment.

Fig. 1o. Bulbus eines jungen Haares am Wurzelhalse bei $b$ geknickt, im weiteren Verlaufe nochmals aus der Wachsthumsrichtung abgelenkt durch narbiges Gewebe bei d. Ringsum die Haaranlage, besonders bei $c$ kleinzellige Infiltration.

Fig. 16. Spitzbogenformige Krümmung einer Haarwurzel. a Huxley'sche, $b$ Henle'sche (innere Wurzel)scheide, $c$ Oberhäntchen des Haares, $d$ Oberhäutchen der äusseren Wurzelscheide, $f, l$ Haarschaft, $g$ Orbicularisbündel, $h$ Rand der Acini einer Meibom'schen Drüse.

Fig. 17 (Hartnack Obj, 2 Oc. 3). Frontalschnitt durch die Lidkante mit einzelnen ,falschen" Haaren a $a$ a, kleinzellige Infiltration der Cutispapillen. Auch höher im Kantengewebe schwăchere entzündliche Infiltration. Längs- und schief getroffene Haare, $b$ welliger Verlauf einer Haarwurzel, $c$ Haarpapille.

Fig. 18 (Hartnack Obj. 4 Oc. 3). Längsschnitt durch ein oberes Augenlid. Injectionspräparat. Das Grundgewebe ungefarbt.

1 Arcus tarseus inferior. 2 Aeste desselben zum Lidrande, aus welchem die Randgefässschlingen (3) für die Cutispapillen der intermarginalen Iidrandfläche hervorgehen. Letztere communiciren vorn über der vorderen Lidkante mit den Hautgefässen 8 , hinten gehen sie direct in die Conjunctivalgefăsse 4 und 5 über. Die Conjunctivalgefässe sind selbstständig, stammen aus den oberen Tarsalgefässen. 10: um den convexen 
Prim. Haarnenbildung auf dex intermarginalen Kantenfāache etc. 91

Knorpelrand zur Conjunctiva gelangende Aeste der oberen Tarsalgefässe. Die Conjunctivalgefässe 10,5 und 4 sind ron den Knorpelgefässen durch die vordere Knorpelfläche bei $f$ völlig getrennt. 6 und 7 praetarseale Gefässe. 9 Netz von Capillaren um die Meibom'schen Drüsen im Knorpel. $11 \mathrm{dgl}$. um eine Cilienhaarwurzel. a Haarpapille mit Gefässschlinge. $b$ Haar senkrecht zur Lidrandfläche gerichtet. $c$ Orbicularisbündel, $d$ abgetrennter Theil: Riolan'scher Muskel, $c$ Acini der Meibom'schen Drüsen $f$. Relativ gefässlose vordere Knorpelftäche zwischen Conjunctiva and Meibom'scher Drüse.

Die Figuren 1, 17 und 18 sind von der kunstfertigen Hand der Herren Lakschewitz und Kiersnowski nach meinen Präparaten gezeichnet worden. 
v. Graefe's Archiv Bd. XXXVII. 2.

Taf. VII.

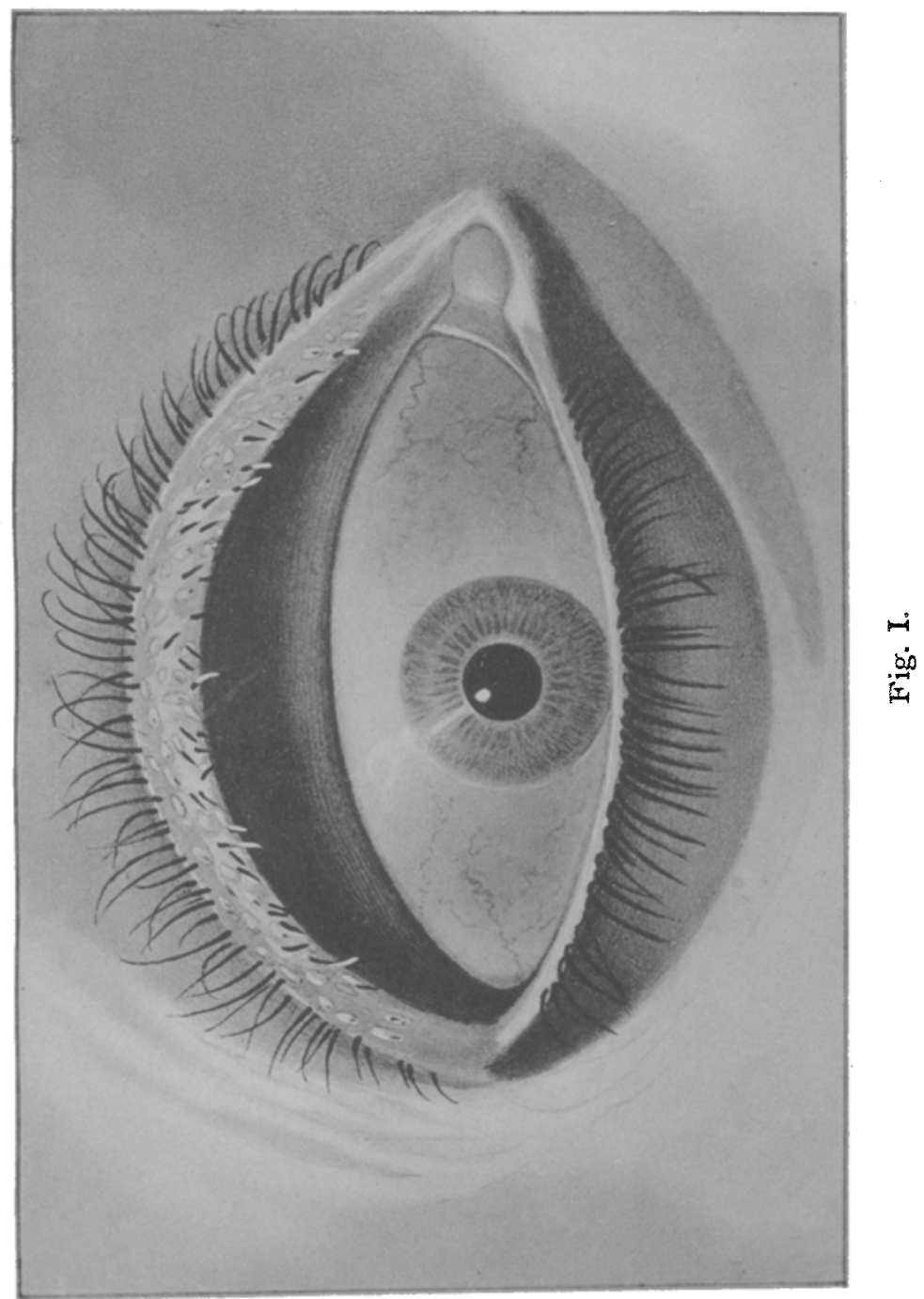

Pinxit $A$. Kiersnowski.

Lichtalutck vor Julus klinhardt, Leipzis.

Verlag fon Wilh. Engelmann in Leipzig. 
v. Graefe's Archiv BdXXXYII2.

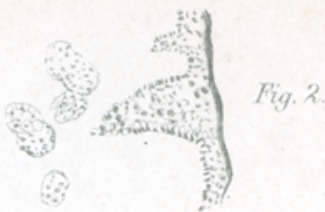

Fig. 4

Fig. 3.

Fig. 5.
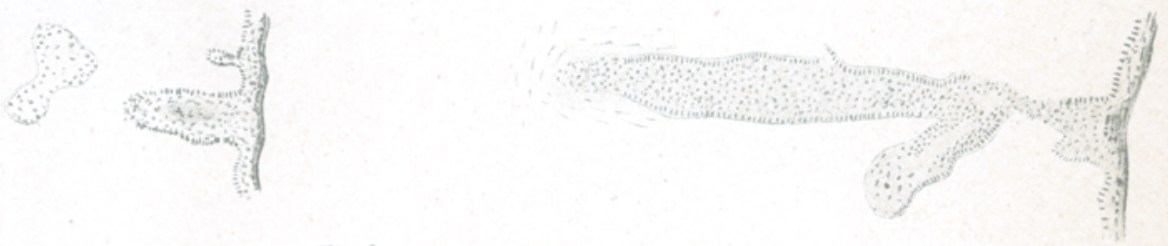

Fig.6.

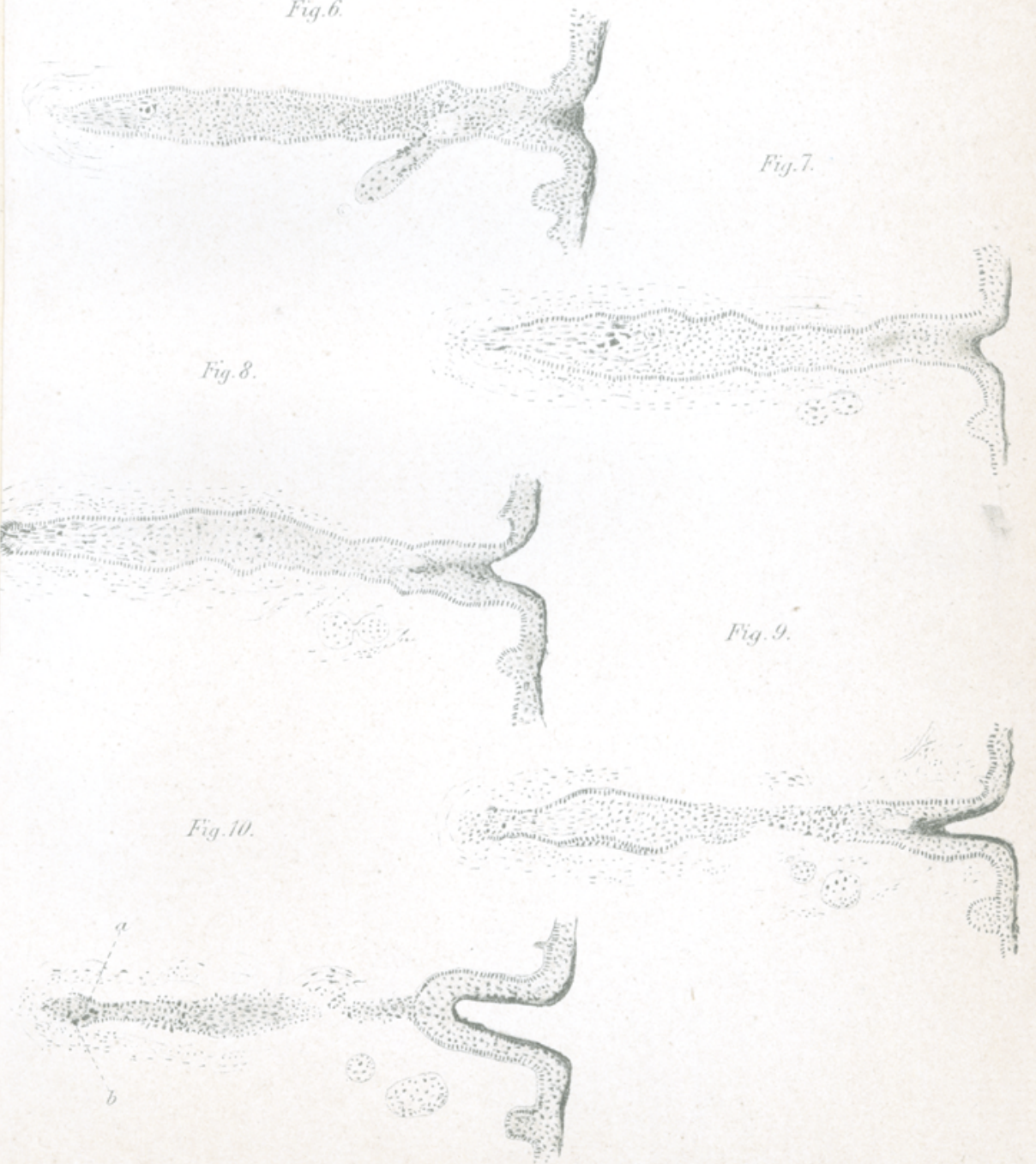


ע. Graefe's Archiw Bd.XXXVII 2.

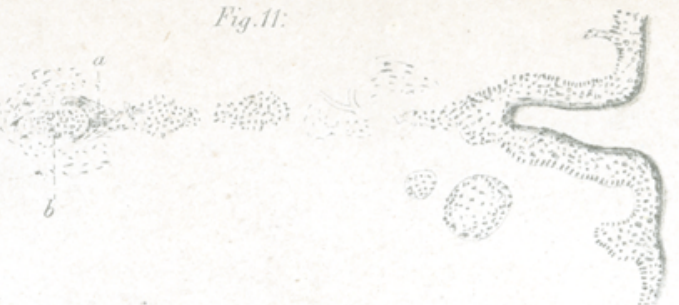

Fig. 12

Fig. 13

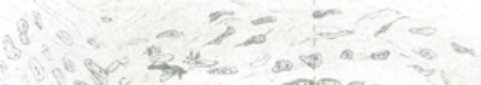

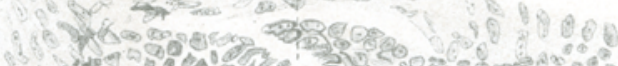

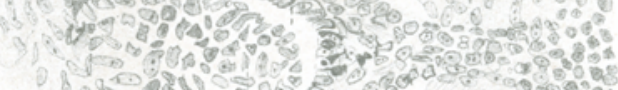

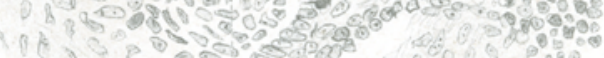

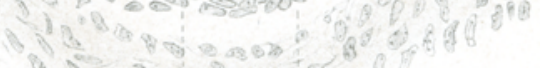

$$
\begin{aligned}
& x^{2}=0,-20,0
\end{aligned}
$$

Fig. 14

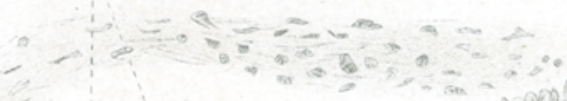

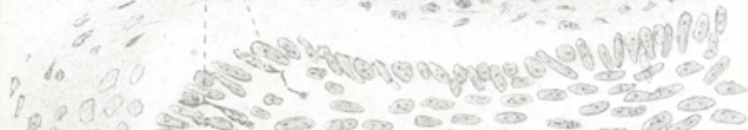

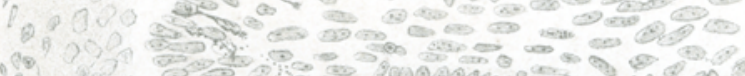
- 13 l a It $x^{3}$ ir

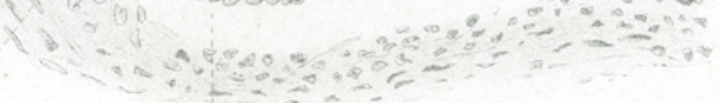

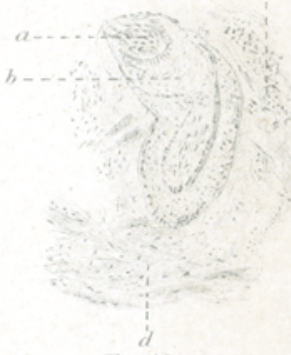

Fig. 16

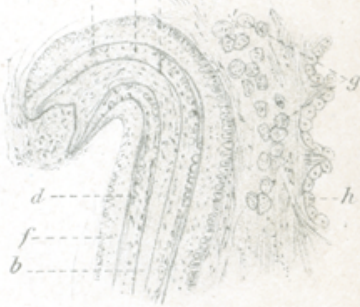

Fig. /7
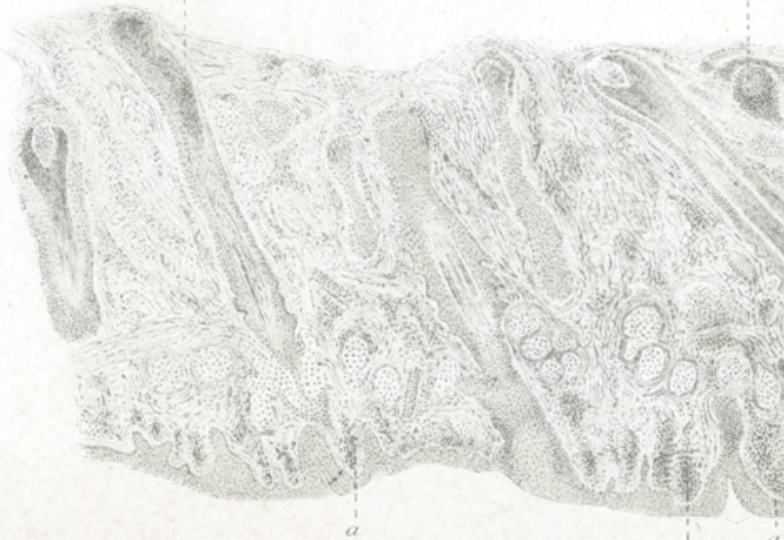
v. Graefe's Archiv Bd. XXYVII. 2.

Fig. 18.

Taf. .

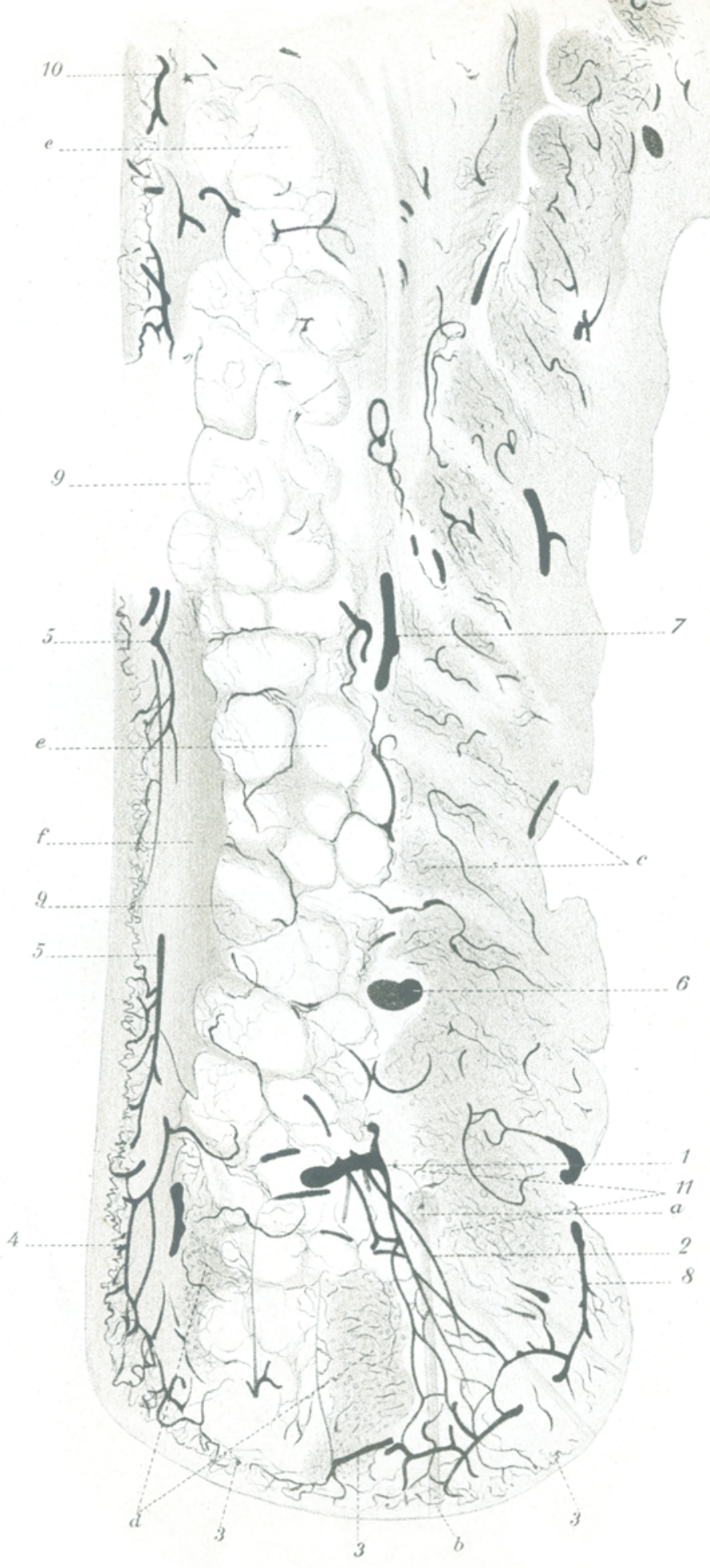

Verlag v Wilh. Engemann in Leip as 